\title{
A Field Theory Model for Dark Matter and Dark Energy in Interaction
}

\author{
Sandro Micheletti ${ }^{1 *}$, Elcio Abdalla ${ }^{1 \dagger}$, Bin Wang ${ }^{2 \ddagger}$ \\ 1 Instituto de Física, Universidade de São Paulo, \\ CP 66318, 05315-970, São Paulo, Brazil and \\ 2 Department of Physics, Fudan University, 200433 Shanghai, China
}

\begin{abstract}
We propose a field theory model for dark energy and dark matter in interaction. Comparing the classical solutions of the field equations with the observations of the CMB shift parameter, BAO, lookback time and Gold supernovae sample, we observe a possible interaction between dark sectors with energy decay from dark energy into dark matter. The observed interaction provides an alleviation to the coincidence problem.

PACS numbers: 98.80.C9; 98.80.-k
\end{abstract}

\footnotetext{
* Email:smrm@fma.if.usp.br

$\dagger$ Email:eabdalla@fma.if.usp.br

$\ddagger$ Email:wangb@fudan.edu.cn
} 
Recently there have been several papers dealing with interacting Dark Energy and Dark Matter [1, 2, 3, 4, 5]. It was argued that Dark Energy and Dark Matter interact via a small coupling, of the order of magnitude of the fine structure constant [6]. Employing some data sets from observational cosmology, including CMB shift parameter, BAO, age parameter and supernovae observations etc., it has been shown that the interacting model is a useful and robust model at the order of one standard deviation [1, 4, 7], while some observations are good enough at two $\sigma$ level, providing some confidence on the results.

Most available discussions on the interaction between dark sectors are concentrated on the phenomenological investigations. It is of great interest to describe the interaction between dark energy and dark matter from a fundamental field theory point of view. Recently, some attempts have been proposed in [13]. In order to follow this thread, we consider now an interacting field theory with two fields describing each of the dark components, a fermionic field for Dark Matter and a bosonic field for the Dark Energy, which here we adopt to be the tachyon field [8]-[11]. We thus consider the Lagrangian

$$
\mathcal{L}=\sqrt{-g}\left\{-V(\varphi) \sqrt{1-\alpha \partial^{\mu} \varphi \partial_{\mu} \varphi}+\frac{i}{2}\left[\bar{\Psi} \gamma^{\mu} \nabla_{\mu} \Psi-\bar{\Psi} \overleftarrow{\nabla}_{\mu} \gamma^{\mu} \Psi\right]-(M-\beta \varphi) \bar{\Psi} \Psi\right\}
$$

where $\alpha$ is a constant with dimension $\mathrm{MeV}^{-4}, \beta$ a coupling between dark energy and dark matter fields, $V(\varphi)$ the tachyonic potential and $g$ the determinant of the metric. For a Friedmann-Robertson-Walker cosmology $g_{\mu \nu}=\operatorname{diag}\left(1,-a(t)^{2},-a(t)^{2},-a(t)^{2}\right)$ one finds the equation of motion for the scalar field to be

$$
\ddot{\varphi}=-\left(1-\alpha \dot{\varphi}^{2}\right)\left[\frac{1}{\alpha} \frac{d \ln V(\varphi)}{d \varphi}+3 H \dot{\varphi}-\frac{\beta \bar{\Psi} \Psi}{\alpha V(\varphi)} \sqrt{1-\alpha \dot{\varphi}^{2}}\right],
$$

with $H=\frac{\dot{a}}{a}$. We also have

$$
\begin{aligned}
& \frac{d\left(a^{3} \Psi^{\dagger} \Psi\right)}{d t}=0 \\
& \frac{d\left(a^{3} \bar{\Psi} \Psi\right)}{d t}=0 .
\end{aligned}
$$

From the latter, $\bar{\Psi} \Psi=\frac{\bar{\Psi}_{0} \Psi_{0} a_{0}^{3}}{a^{3}}$. We note that such a result follows from the homogeneity assumed for Dark Matter distribution. Thus, Dark Matter in our model just follows the 
universe expansion, what is consistent with the cosmological principle. Moreover,

$$
\begin{aligned}
\rho_{\varphi} & =\frac{V(\varphi)}{\sqrt{1-\alpha \dot{\varphi}^{2}}}, \\
P_{\varphi} & =-V(\varphi) \sqrt{1-\alpha \dot{\varphi}^{2}}, \\
\rho_{\Psi} & =M^{*} \bar{\Psi} \Psi \\
P_{\Psi} & =0
\end{aligned}
$$

where we defined the effective mass $M^{*} \equiv M-\beta \varphi$. Note that $\omega_{\varphi} \equiv P_{\varphi} / \rho_{\varphi}=-\left(1-\alpha \dot{\varphi}^{2}\right)$. Deriving 5 and 7 with respect to time and using 2 and 4 , we get

$$
\begin{aligned}
\dot{\rho_{\varphi}}+3 H \rho_{\varphi}\left(\omega_{\varphi}+1\right) & =\beta \dot{\varphi} \frac{\bar{\Psi}_{0} \Psi_{0} a_{0}^{3}}{a^{3}} \\
\dot{\rho_{\Psi}}+3 H \rho_{\Psi} & =-\beta \dot{\varphi} \frac{\bar{\Psi}_{0} \Psi_{0} a_{0}^{3}}{a^{3}} .
\end{aligned}
$$

These equations are very similar to those usually used as a phenomenological model for the interaction between dark matter and dark energy [4, 7, 12]. The right hand side in the above equations does not contain the Hubble parameter $H$ explicitly, but it does contain the time derivative of the scalar field, which should behave as the inverse of the cosmological time, replacing thus the Hubble parameter in the phenomenological models.

The Friedmann equation for a flat universe reads

$$
H^{2}=\frac{1}{3 M_{p l}^{2}}\left[M^{*} \frac{\bar{\Psi}_{0} \Psi_{0} a_{0}^{3}}{a^{3}}+\frac{V(\varphi)}{\sqrt{1-\alpha \dot{\varphi}^{2}}}\right],
$$

where $M_{p l}^{2}=(8 \pi G)^{-1}$ e $H=\frac{\dot{a}}{a}$.

Some analytic solutions in the pure bosonic case have been found in [9] and [11] for the potential

$$
V(\varphi)=\frac{m^{4+n}}{\varphi^{n}}, n>0 .
$$

We choose, at this moment, $n=2$, which leads to a power law expansion of the universe. However, we shall see that this choice is really not important and some properties depend little on the actual choice of $n$. This actually lowers the appeal of the present model.

Let us now compare the interacting DE with the observational data. We will compare the interacting tachyonic model with the luminosity distance of the Gold supernova sample (182 type Ia supernovae observations), the shift parameter of CMB radiation, the measurement of the Baryonic Acoustic Oscillations (BAO) and ages of galaxy clusters (see [7, 13, 14, 15, 16]). 
It is convenient to rewrite (2) in terms of two first order equations. Using $V(\varphi)$ as in (12) with $n=2$, (2) becomes

$$
\begin{aligned}
& \dot{\omega}=-\frac{4 \omega \sqrt{\omega+1}}{\phi}+6 H \omega(\omega+1)-\frac{2 \beta \bar{\Psi}_{0} \Psi_{0} a_{0}^{3}}{\alpha \sqrt{\alpha} m^{6}} \frac{\omega \sqrt{|\omega|(\omega+1)} \phi^{2}}{a^{3}}, \\
& \dot{\phi}=\sqrt{\omega+1} .
\end{aligned}
$$

Above, we defined $\varphi \equiv \frac{\phi}{\sqrt{\alpha}}$. Equating the actual values of $\rho_{\Psi}$ and $\rho_{\phi}$ with the observed values, $\rho_{\Psi_{0}}=3 M_{p l}^{2} H_{0}^{2} \Omega_{\Psi_{0}}$ and $\rho_{\phi_{0}}=3 M_{p l}^{2} H_{0}^{2}\left(1-\Omega_{\Psi_{0}}\right)$, we can replace $\bar{\Psi}_{0} \Psi_{0}$ and $m$ by observable quantities: $M \bar{\Psi}_{0} \Psi_{0}=3 M_{p l}^{2} H_{0}^{2} \Omega_{\Psi_{0}} /\left(1-\frac{\beta}{M} \frac{\phi_{0}}{\sqrt{\alpha}}\right)$ and $\alpha m^{6}=3 M_{p l}^{2} H_{0}^{2}\left(1-\Omega_{\Psi_{0}}\right) \phi_{0}^{2} \sqrt{\left|\omega_{0}\right|}$. Therefore,

$$
\begin{aligned}
\frac{d \omega}{d z} & =\frac{4 \omega \sqrt{\omega+1}}{H_{0} E(z) \phi(1+z)}-\frac{6 \omega(\omega+1)}{1+z} \\
& +\frac{2\left(\frac{\beta}{M}\right) \frac{\Omega_{\Psi_{0}}}{\sqrt{\alpha}}}{\left(1-\Omega_{\Psi_{0}}\right)\left(1-\frac{\beta}{M} \frac{\phi_{0}}{\sqrt{\alpha}}\right)} \frac{\omega \sqrt{\left|\frac{\omega}{\omega_{0}}\right|(\omega+1)}\left(\frac{\phi}{\phi_{0}}\right)^{2}}{H_{0} E(z)}(1+z)^{2}, \\
\frac{d \phi}{d z} & =-\frac{\sqrt{\omega+1}}{H_{0} E(z)(1+z)}, \\
\frac{d t}{d z} & =-\frac{1}{(1+z) H_{0} E(z)}
\end{aligned}
$$

where $H_{0}=2.133 \mathrm{~h} \times 10^{-39} \mathrm{MeV}$ is the value of the Hubble parameter today, $\Omega_{\Psi_{0}}=\frac{\rho_{\Psi_{0}}}{3 M_{p l}^{2} H_{0}^{2}}$ and

$$
E(z)=\sqrt{\frac{1-\frac{\beta}{M} \frac{\phi}{\sqrt{\alpha}}}{1-\frac{\beta}{M} \frac{\phi_{0}}{\sqrt{\alpha}}} \Omega_{\Psi_{0}}(1+z)^{3}+\left(\frac{\phi_{0}}{\phi}\right)^{2} \sqrt{\mid \frac{\omega_{0}}{\omega}} \mid\left(1-\Omega_{\Psi_{0}}\right) .}
$$

The parameter $\alpha$ is fixed as $\sqrt{\alpha}=1.607 \times 10^{39} \mathrm{MeV}^{-2} \sim\left(H_{0} \times M e V\right)^{-1}$. Since $\phi_{0} \sim H_{0}^{-1}$ [9] [10], such $\alpha$ was chosen such that the last term in (15) is of the same order of magnitude as the other terms. In fact, we shall see - and it is easy to infer from the above equations - that only $\frac{\beta}{M \sqrt{\alpha}}$ can be obtained, that is, $\alpha$ and $M$ can be absorbed in the redefinition of $\beta$. Thus we have, as parameters of the model, $\left(\frac{\beta}{M \sqrt{\alpha}}, \phi_{0}, h, \Omega_{\Psi_{0}}, \omega_{0}\right)$.

In [17], the lookback time method was discussed, allowing to use the cluster age to fix the parameters. Given an object $i$ at redshift $z_{i}$, its age $t\left(z_{i}\right)$ is defined as the difference between the age of the universe at $z_{i}$ and the age of the universe at the formation redshift of the object, $z_{F}$, that is,

$$
\begin{aligned}
t\left(z_{i}\right)= & H_{0}^{-1}\left[\int_{z_{i}}^{\infty} \frac{d z^{\prime}}{\left(1+z^{\prime}\right) E\left(z^{\prime}\right)}-\int_{z_{F}}^{\infty} \frac{d z^{\prime}}{\left(1+z^{\prime}\right) E\left(z^{\prime}\right)}\right] \\
= & H_{0}^{-1} \int_{z_{i}}^{z_{F}} \frac{d z^{\prime}}{\left(1+z^{\prime}\right) E\left(z^{\prime}\right)}=t_{L}\left(z_{F}\right)-t_{L}\left(z_{i}\right)
\end{aligned}
$$


where $t_{L}$ is the lookback time given by

$$
t_{L}(z)=H_{0}^{-1} \int_{0}^{z} \frac{d z^{\prime}}{\left(1+z^{\prime}\right) E\left(z^{\prime}\right)} .
$$

Using (19), the observational lookback time $t_{L}^{\text {obs }}\left(z_{i}\right)$ is

$$
\begin{array}{r}
t_{L}^{o b s}\left(z_{i}\right)=t_{L}\left(z_{F}\right)-t\left(z_{i}\right)=\left[t_{0}^{o b s}-t\left(z_{i}\right)\right]-\left[t_{0}^{o b s}-t_{L}\left(z_{F}\right)\right] \\
=t_{0}^{o b s}-t\left(z_{i}\right)-d f
\end{array}
$$

where $t_{0}^{o b s}$ is the estimated age of the universe today and $d f$ is the delay factor,

$$
d f \equiv t_{0}^{o b s}-t_{L}\left(z_{F}\right)
$$

We now minimize $\chi_{l b t}^{2}$,

$$
\chi_{l b t}^{2}=\sum_{i=1}^{N} \frac{\left[t_{L}\left(z_{i}, \vec{p}\right)-t_{L}^{o b s}\left(z_{i}\right)\right]^{2}}{\sigma_{i}^{2}+\sigma_{t_{0}^{o b s}}^{2}},
$$

where $t_{L}\left(z_{i}, \vec{p}\right)$ is the theoretical value of the lookback time in $z_{i}, \vec{p}$ denotes the theoretical parameters, $t_{L}^{\text {obs }}\left(z_{i}\right)$ is the corresponding observational value given by (21), $\sigma_{i}$ is the uncertainty in the estimated age $t\left(z_{i}\right)$ of the object at $z_{i}$, which appears in (21) and $\sigma_{t_{0}^{o b s}}$ is the uncertainty in getting $t_{0}^{o b s}$. The delay factor $d f$ appears because of our ignorance about the redshift formation $z_{F}$ of the object and has to be adjusted. Note, however, that the theoretical lookback time does not depend on this parameter, and we can marginalize over it.

In [18] and [19] the ages of 35 and 32 red galaxies are respectively given. For the age of the universe one can adopt $t_{0}^{o b s}=13.73 \pm 0.12$ Gyr [20]. Although this estimate for $t_{0}^{\text {obs }}$ has been obtained assuming a $\Lambda C D M$ universe, it does not introduce systematical errors in our calculation: any systematical error eventually introduced here would be compensated by the adjust of $d f$, in (21). On the other hand, this estimate is in perfect agreement with other estimates, which are independent of the cosmological model, as for example $t_{0}^{o b s}=12.6_{-2.4}^{+3.4} \mathrm{Gyr}$, obtained from globular cluster ages [21] and $t_{0}^{\text {obs }}=12.5 \pm 3.0 \mathrm{Gyr}$, obtained from radioisotopes studies [22].

For the cosmic radiation shift parameter in the flat universe we have

$$
R=\sqrt{\Omega_{M}} \int_{0}^{z_{l s}} \frac{d z^{\prime}}{E\left(z^{\prime}\right)},
$$


where $z_{l s}=1089$ is the last scattering surface redshift parameter. The value $R$ has been estimated in [23] from the 3-years WMAP [24] results as $R_{o b s}=1.70 \pm 0.03$, for the flat universe, and is very weakly model dependent. Thus

$$
\chi_{C M B}^{2}=\frac{\left[R-R_{o b s}\right]^{2}}{\sigma_{R}^{2}} .
$$

Baryonic Acoustic Oscilations (BAO) [25] is described in terms of the parameter

$$
A=\sqrt{\Omega_{M}} E\left(z_{B A O}\right)^{-1 / 3}\left[\frac{1}{z_{B A O}} \int_{0}^{z_{B A O}} \frac{d z^{\prime}}{E\left(z^{\prime}\right)}\right]^{2 / 3}
$$

where $z_{B A O}=0.35$. It has been estimated that $A_{o b s}=0.469\left(\frac{n_{s}}{0.98}\right)^{-0.35} \pm 0.017$, with $n_{s}=0.95$ [24] being the scalar spectral index. We thus add to $\chi^{2}$ the term

$$
\chi_{B A O}^{2}=\frac{\left[A-A_{o b s}\right]^{2}}{\sigma_{A}^{2}} .
$$

Finally, we add the 182 supernovae data from SNLS [26], recent supernovae from HST/GOODS and further old data, as compiled by Riess, et. al. [27]. Defining the distance modulus

$$
\mu(z)=5 \log _{10}\left[c(1+z) \int_{0}^{z} \frac{d z^{\prime}}{E\left(z^{\prime}\right)}\right]+25-5 \log _{10} H_{0}
$$

we have the contribution

$$
\chi_{S N}^{2}=\sum_{j=1}^{182} \frac{\left[\mu\left(z_{j}\right)-\mu_{o b s}\left(z_{j}\right)\right]^{2}}{\sigma_{j}^{2}}
$$

We use que expression

$$
\chi^{2}=\chi_{S N}^{2}+\chi_{B A O}^{2}+\chi_{C M B}^{2}+\chi_{l b t}^{2}+\frac{\left(h-h_{o b s}\right)^{2}}{\sigma_{h}^{2}}+\frac{\left(\Omega_{\Psi_{0}}-\Omega_{M o b s}\right)^{2}}{\sigma_{\Omega_{M}}^{2}},
$$

where the last two terms correspond to gaussian priors for $h$ [28] and $\Omega_{\Psi_{0}}$ [27], respectively: $h_{o b s}=0.72 \pm 0.08$ and $\Omega_{\text {Mobs }}=0.28 \pm 0.04$.

The likelihood function is given by

$$
\mathcal{L}\left(\frac{\beta}{M \sqrt{\alpha}}, \phi_{0}, h, \Omega_{\Psi_{0}}, \omega_{0}\right) \propto \exp \left[-\frac{\chi^{2}\left(\frac{\beta}{M \sqrt{\alpha}}, \phi_{0}, h, \Omega_{\Psi_{0}}, \omega_{0}\right)}{2}\right] .
$$

We present in the table the individual (marginalized) best fit for each parameter, with respective deviations. Figure (1a) shows the curve $\mu(z)$, corresponding to the global best fit. Figure $(1 \mathrm{~b})$ shows the fit of the lookback time $t_{L}(z)$. 
Table 1: Values of the model parameters from lookback time, BAO, CMB and SNe Ia.

\begin{tabular}{|c|c|}
\hline$h$ & $0.631 \pm 007 \pm 0.015 \pm 0.022$ \\
\hline$\Omega_{\Psi_{0}}$ & $0.324 \pm 0.017 \pm 0.033 \pm 0.050$ \\
\hline$\omega_{0}$ & $-0.979_{-0.003-0.013-0.018}^{+0.106+0.227+0.283}$ \\
\hline$\phi_{0} H_{0}$ & $\phi_{0} H_{0}>1.3(2 \sigma)$ \\
\hline$\frac{\beta}{M \sqrt{\alpha} H_{0}}$ & $\frac{\beta}{M \sqrt{\alpha} H_{0}}<0.071(2 \sigma)$ \\
\hline
\end{tabular}
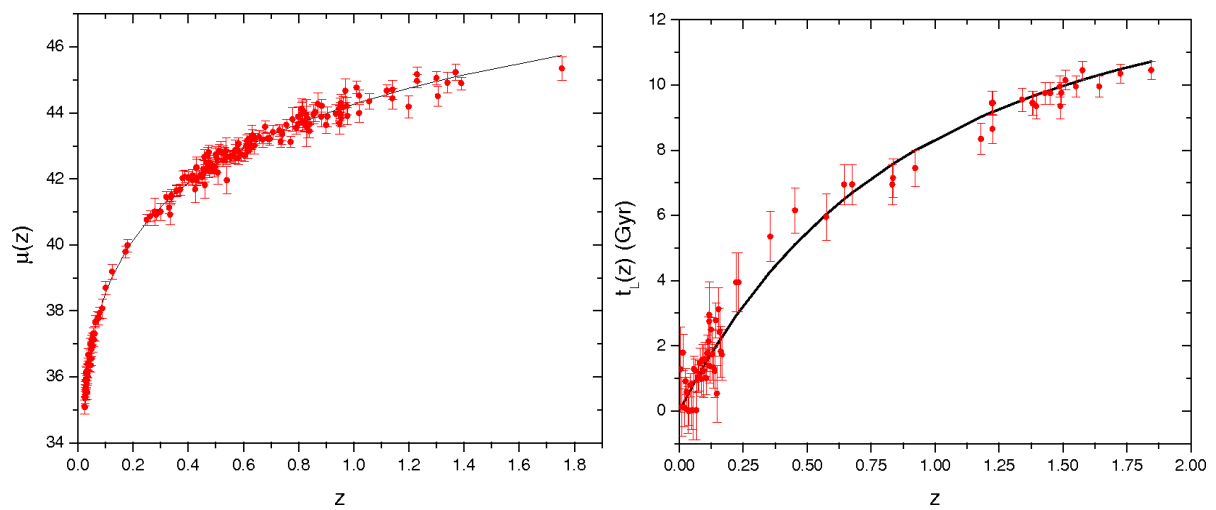

FIG. 1: Results corresponding to the global best fit (lookback time + CMB + BAO + SNe Ia).a) Theoretical distance modulus compared to $182 \mathrm{SNe}$ Ia data. b) Theoretical $t_{L}(z)$ versus 67 galaxy clusters data.

The parameters $\phi_{0}$ and $\frac{\beta}{M \sqrt{\alpha}}$ are strongly degenerated. Indeed, in figure 2 we see that the effect of both parameters are the same on the densities of Dark Energy and Dark Matter: increasing $\phi_{0}$ is equivalent to decreasing $\frac{\beta}{M \sqrt{\alpha}}$. Here, it would be convenient to observe that, even in the non interacting case (when $\frac{\beta}{M \sqrt{\alpha}}=0$ ), as we can see in figure $2 \mathrm{~b}$, the $\Omega_{\Psi}$ never goes to one. Rather, the ratio of the Dark Matter to Dark Energy densities remain constant in the Dark Matter domination era, because, in this era, the equation of state parameter of the Dark Energy approaches zero, and thus Dark Energy behaves as Dark Matter in this period - this feature had already been underlined in [10]. Therefore, in the Tachyonic Dark Energy model the coincidence problem is less serious. However, the ratio of the Dark Matter to Dark Energy densities depends on the parameters of the model. In particular, lower values of $\phi_{0}$ turns this ratio higher. The introduction of the coupling furnishes an additional improvement in the coincidence problem, diminishing the Dark Matter to Dark 
Energy ratio, if the coupling constant $\frac{\beta}{M \sqrt{\alpha}}$ is negative, as we can see in figure 2a. Positive values of $\frac{\beta}{M \sqrt{\alpha}}$, on the other hand, aggravates such a problem.
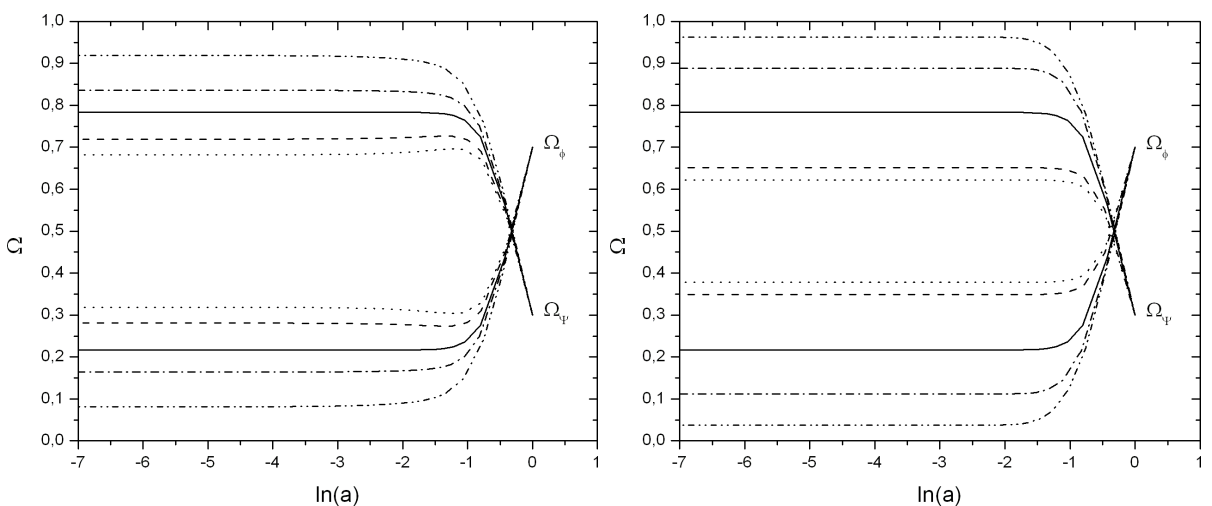

FIG. 2: Relative densities of Dark Energy and Dark Matter, $\Omega_{\phi}$ and $\Omega_{\Psi}$, as functions of the scale factor $a$. a) for $\phi_{0}$ constant $\left(\phi_{0} H_{0}=2.5\right)$. The dot-dot-dashed, dot-dashed, solid, dashed and dotted lines are for $\frac{\beta}{M \sqrt{\alpha} H_{0}}=+0.125, \frac{\beta}{M \sqrt{\alpha} H_{0}}=+0.0625, \frac{\beta}{M \sqrt{\alpha} H_{0}}=0, \frac{\beta}{M \sqrt{\alpha} H_{0}}=-0.125$ and $\frac{\beta}{M \sqrt{\alpha} H_{0}}=-0.25$, respectively. b) for $\frac{\beta}{M \sqrt{\alpha}}$ constant $\left(\frac{\beta}{M \sqrt{\alpha}}=0\right)$. The dot-dot-dashed, dot-dashed, solid, dashed and dotted lines are for $\phi_{0} H_{0}=1.8, \phi_{0} H_{0}=2.0, \phi_{0} H_{0}=2.5, \phi_{0} H_{0}=5.0$ and $\phi_{0} H_{0}=7.5$, respectively.

In order to compare the model with our previous predictions [4, 6, 7, 12] we compute the likelihood functions concerning the various parameters of our model. Our main previous prediction concerns the behaviour of the interaction, especially its sign. As it turns out, the model is very degenerated, but most of the allowed values of $\beta$ are consistent with a negative coupling.

In figure 3 we plot the behaviour of the $\beta$ versus $\phi_{0}$ contour for $1 \sigma$ and $2 \sigma$. Since the diagram is unbounded for negative $\beta$, its allowed values are, generally speaking, negative, although we cannot rule out a small positive coupling. The likelihood of $\beta$ marginalizing all the other parameters is not normalizable, being consistent with all values of $\frac{\beta}{M \sqrt{\alpha}}$ below a small positive value. Most of the allowed values are negative, see figure 4 . These results indicate that if there is a coupling connecting the dark sectors, it is more probable for the dark energy to decay into dark matter, which is consistent with the fact obtained in the study of thermodynamics [29].

In figure 5, the first diagram concerns the Dark Matter fraction $\Omega_{\Psi_{0}}$ versus $\frac{\beta}{M \sqrt{\alpha}}$ contours. We see that the observed value holds almost independent of $\frac{\beta}{M \sqrt{\alpha}}$ if this latter is not large 
positive. Similar conclusions can be drawn for the diagram of the Hubble constant compared to $\phi_{0}$ as well as Dark Matter versus $\phi_{0}$. The likelihood of $\phi_{0}$ is shown in figure 6 .

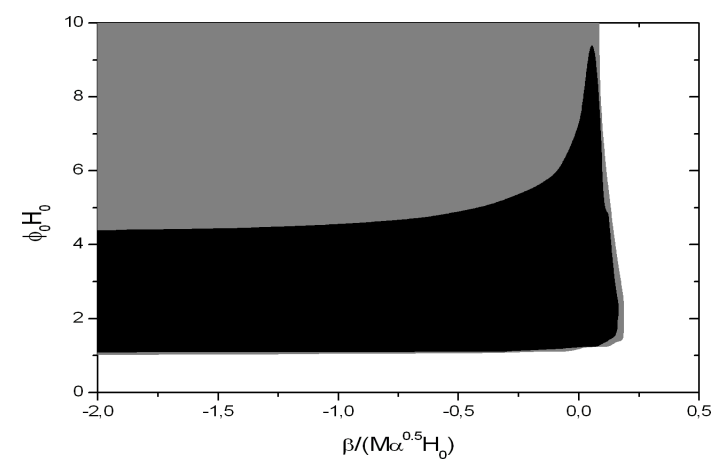

FIG. 3: Two dimensional distribution of $\beta$ and $\phi_{0}$ ( $1 \sigma$ and $2 \sigma$ contours). Notice that there is a strong degeneracy. $\beta$ can go to arbitrarily large negative values. For positive values the function decays quickly to zero. The expectation of $\beta$ cannot be computed due to the fact that the distribution does not approach zero. Thus, $\beta$ should most probably be negative.

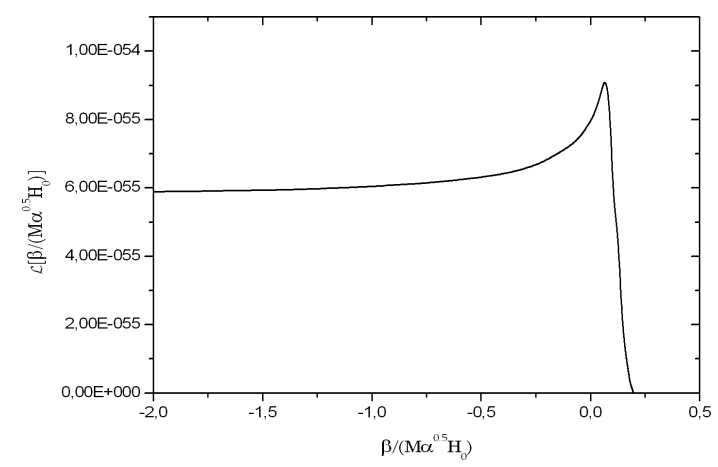

FIG. 4: The $\beta$ likelihood function shows a behavior confirming the speculations arising in the previous figure. It is most probable that it is negative.

In order to further understand this problem we consider the consequence of our formulation for an effective fluid interaction such as the one considered in several previous papers $[1,4,6,6]$. In those works, the interaction term in the fluid conservation equations is of the form $\delta H \rho$, where $\delta$ is the coupling constant. After some simple manipulations and using the 

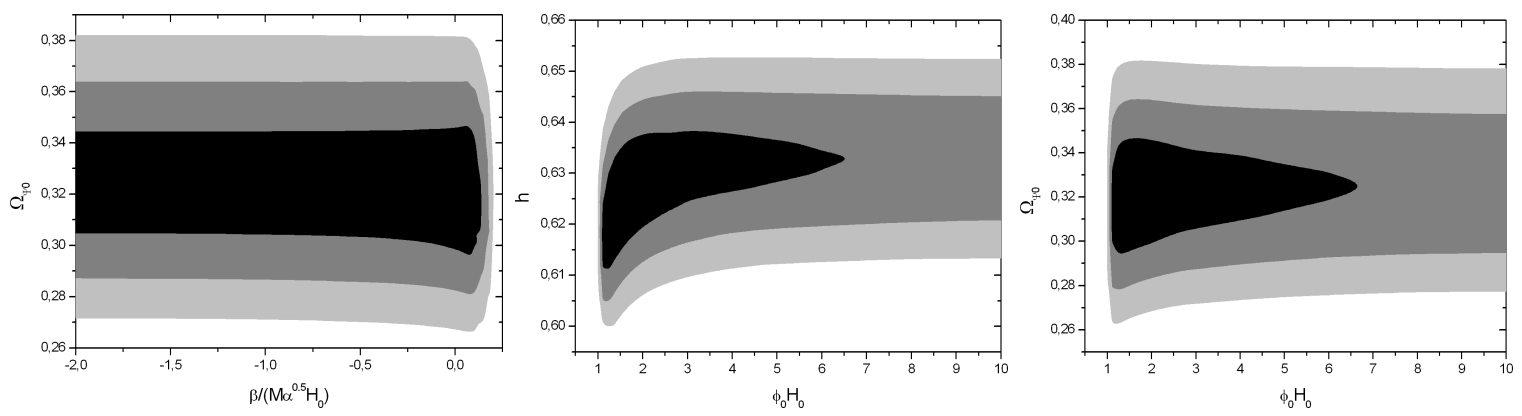

FIG. 5: Two dimensional curves displaying the probability distributions of $\beta$ versus $\Omega_{\Psi_{0}}$, $\phi_{0}$ versus $h$ and $\phi_{0}$ versus $\Omega_{\Psi_{0}}$, respectively.

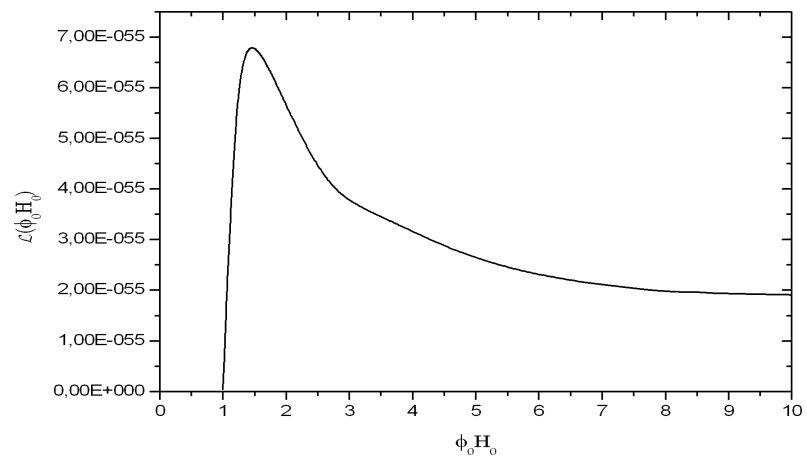

FIG. 6: The $\phi_{0}$ likelihood function.

definition of $w$, the equations (9) and (10) can be put in the form

$$
\begin{aligned}
\dot{\rho_{\varphi}}+3 H \rho_{\varphi}(\omega+1) & =\frac{\frac{\beta}{M \sqrt{\alpha}}}{1-\frac{\beta}{M \sqrt{\alpha}} \phi_{0}} \sqrt{\omega+1} \rho_{\Psi 0}(1+z)^{3}, \\
\dot{\rho_{\Psi}}+3 H \rho_{\Psi} & =-\frac{\frac{\beta}{M \sqrt{\alpha}}}{1-\frac{\beta}{M \sqrt{\alpha}} \phi_{0}} \sqrt{\omega+1} \rho_{\Psi 0}(1+z)^{3},
\end{aligned}
$$

where $\rho_{\Psi_{0}}=3 M_{p l}^{2} H_{0}^{2} \Omega_{\Psi_{0}}$. As we mentioned above, $\sqrt{\alpha} \sim H_{0}^{-1}$. Moreover, for $z>0$, $\omega$ rapidly approaches very small values, so the interaction term in the r.h.s. of (32) and (33) will be of order of $\left(\frac{\frac{\beta}{M}}{1-\frac{\beta}{M \sqrt{\alpha}} \phi_{0}}\right) H_{0} \rho_{\Psi 0}(1+z)^{3}$, very similar to the phenomenological interaction. Therefore, the phenomenological coupling constant $\delta$ would be constituted of two theoretical parameters: $\frac{\beta}{M \sqrt{\alpha}}$ and $\phi_{0}$.

On the other hand, notice that, in the model considered here, the parameter $\delta \equiv \frac{\frac{\beta}{M \sqrt{\alpha}}}{1-\frac{\beta}{M \sqrt{\alpha}} \phi_{0}}$, is in fact an effective coupling constant. This appears in (32) and (33) and in the last term of (15). The only other place where $\frac{\beta}{M \sqrt{\alpha}}$ appears is in (18), but in fact, $H$ is not much 
affected by the coupling, since $\phi$ decreases rapidly to a certain value (typically about $0.8 \phi_{0}$ ), thereafter remaining constant (remember that we are integrating the equations "backward"). Therefore, $\frac{1-\frac{\beta}{M \sqrt{\alpha}} \phi}{1-\frac{\beta}{M \sqrt{\alpha}} \phi_{0}} \simeq 1$.

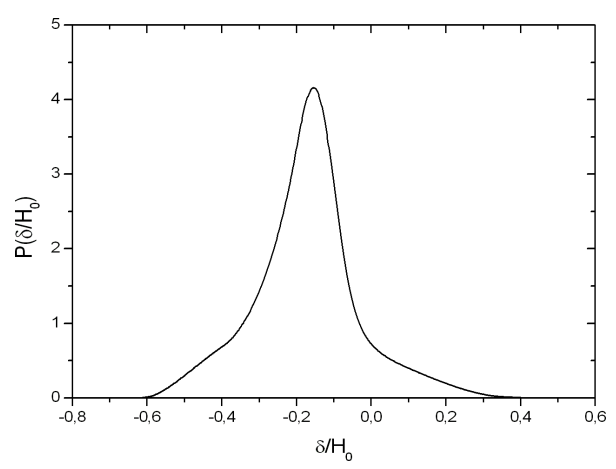

FIG. 7: The likelihood function (34).

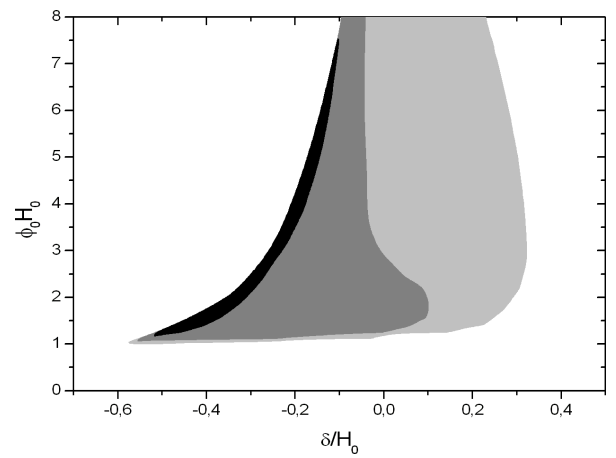

FIG. 8: Two dimensional curves displaying the probability distribution of $\delta$ versus $\phi_{0}$.

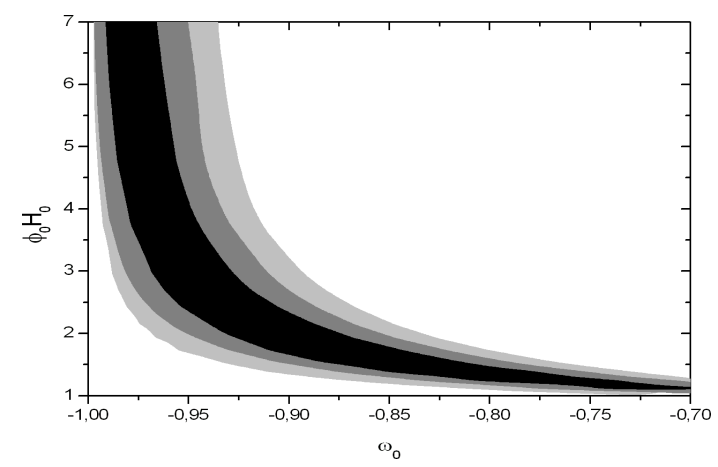

FIG. 9: Two dimensional curve showing the behaviour of $\phi_{0}$ and $w$. 


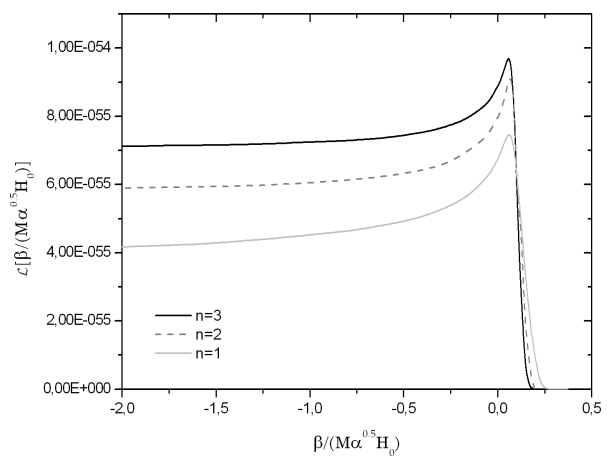

FIG. 10: Dependence of the model on the value of $n$.

We compute now the likelihood of the function $\delta \equiv \frac{\frac{\beta}{M \sqrt{\alpha}}}{1-\frac{\beta}{M \sqrt{\alpha}} \phi_{0}}$. The likelihood of $\delta$ is determined from the likelihoods of $\frac{\beta}{M \sqrt{\alpha}}$ and $\phi_{0}$ accordingly to

$$
P\left(u^{\prime}\right)=\iint \delta\left(u^{\prime}-\delta\right) P\left(\frac{\beta}{M \sqrt{\alpha}}, \phi_{0}\right) d\left(\frac{\beta}{M \sqrt{\alpha}}\right) d \phi_{0} \quad,
$$

where $P\left(\frac{\beta}{M \sqrt{\alpha}}, \phi_{0}\right) \sim \operatorname{prior}\left(\frac{\beta}{M \sqrt{\alpha}}, \phi_{0}\right) \mathcal{L}\left(\frac{\beta}{M \sqrt{\alpha}}, \phi_{0}\right)$ for some prior we choose for $\frac{\beta}{M \sqrt{\alpha}}$ and $\phi_{0}$. We find the result shown in figure 7 . It is convenient to introduce some remarks.

As previously mentioned, we had rewritten $\bar{\Psi}_{0} \Psi_{0}$ in terms of observable quantities, $\bar{\Psi}_{0} \Psi_{0}=\frac{3 M_{p l}^{2} H_{0}^{2} \Omega_{\Psi_{0}}}{M\left(1-\frac{\beta}{M \sqrt{\alpha}} \phi_{0}\right)}$. Since $\bar{\Psi}_{0} \Psi_{0}$ is the number density of particles of Dark Matter, it must be positive. Thus we must have $\frac{\beta}{M \sqrt{\alpha}} \phi_{0}<1$. This constraint has to be included in the prior on $\phi_{0}$ and $\frac{\beta}{M \sqrt{\alpha}}$. Moreover, as a consequence, the effective coupling $\delta$ has the same sign as $\beta$ and more negative (positive) values of $\delta$ are equivalent to more negative (positive) values of $\beta$, turning the ratio of the Dark Matter to Dark Energy lower (higher) in the Dark Matter domination era. Thus, $\delta$ has the same degeneracy with $\phi_{0}$ as $\beta$. However, $\delta$ is much more restricted than $\beta$, being contained in a small interval, as shown in figures 7 and 8 . The constraint $\frac{\beta}{M \sqrt{\alpha}} \phi_{0}<1$ is equivalent to $\phi_{0}<-\frac{1}{\delta}$, for $\delta<0$, as it can be seen if we eliminate $\frac{\beta}{M \sqrt{\alpha}}$ in favor of $\delta$ in the expression for $\bar{\Psi}_{0} \Psi_{0}$. Such a constraint can be clearly seen in figure 8. The maximum of $P(\delta)$, figure 7 , depends on the prior on $\frac{\beta}{M \sqrt{\alpha}}$ and (heavily) on the prior on $\phi_{0}$, but the integrated probability for $\delta<0$ is very weakly prior dependent. We have for the probability for negative coupling $P(\delta<0)=90 \%$. Thus our result is consistent with a small negative value of $\delta$, which implies in DE decaying into DM, alleviating the coincidence problem.

We also learn, in this connection, that in this model a more negative equation of state 
for Dark Energy is connected with a larger value of $\phi_{0}$ as shown in figure 9 . However, such a result is rather model dependent.

The first attempt of this study was to describe a few details about the Dark Energy and Dark Matter behaviours and thus we chose the model by specifying the index $n$ to be 2 . However, in figure 10 we see that the numerical solution shows nothing extraordinary for such a choice of $n$. Indeed, for $n=1,2,3$ the results of the likelihood of the coupling $\beta$ are surprisingly similar.

All $n$ tested in this work (until $n=10$ ) were capable to reproduce the features of the observed universe (at least, of the background), and, in fact, the solutions of the equation of motion for all $n$ tested had the same qualitative behavior: it reproduced the actual period of accelerated expansion, driven by the Dark Energy domination, and the Dark Energy equation of state parameter approaching zero in the Dark Matter domination era, forcing the ratio $\rho_{\Psi} / \rho_{\phi}$ to be a constant in this era. In [11] two approximate solutions had been found, valid for a tachyon dominated universe: one which corresponds to $\omega \approx-1$, for $0<n<2$ and another which corresponds to $\omega \approx 0$, for $n>2$. The numerical solutions for the exact equation of motion, encountered by us, in fact reproduce these predicted behaviour, but only asymptotically, in the far future $(a(t)>>1)$, when $\Omega_{\phi} \rightarrow 1$. These results are consistent with those found in ref [13], namely a dynamical attractor behaviour for general values of $n$.

The model has further features that we consider being drawbacks, as e.g. the fact that $w>-1$ or also the extreme non linearity of the action, rendering the calculation clumsy and the particle interpretation unclear. From a positive side, the problem can be opened up for more realistic models of Quantum Field Theory, as in [30].

In spite of the simplicity of the model, the comparison of the model with the values of cosmological parameters leads to the conclusion that the interaction is consistent with the observations at least at one standard deviation, possibly at two, for negative coupling. This encourages us to look for more sophisticated theoretical field models for the explanation of the Dark Matter and Dark Energy behaviors as well as their origins. In particular, the present model does not account for equations of state with $\omega<-1$. We could try to mimic this fact by taking more sophisticated potentials $(V<0)$ or a larger number of fields, but that would enlarge the number of parameters of the model. Since a transition redshift is not yet well established we prefer to stay with the more conservative case.

A further point we have not dealt with is the comparison with the structure formation. 
The procedure might imply further (and better) constraints for $\beta$ and $\phi_{0}$. However, we did not find it worthwhile pursuing further this simple model and we left aside this possibility.

As a conclusion we can state that it is reasonable to expect that DE and DM interact via a small but calculable and observable coupling, possibly giving an alternative to the usual cosmological constant explanation of Dark Energy.

Acknowledgements: This work has been supported by FAPESP and CNPQ of Brazil, by NNSF of China, Shanghai Education Commission, and Shanghai Science and Technology Commission.

[1] W. Zimdahl and D. Pavon Phys. Lett. B521 (2001) 133; L. P. Chimento, A. S. Jakubi, D. Pavon, W. Zimdahl Phys. Rev. D67 (2003) 083513.

[2] Rogerio Rosenfeld Phys. Rev. D75 (2007) 083509; M. Quartin, M. O. Calvao, S. E. Joras, R.R. Reis, I. Waga JCAP 0805 (2008) 007; Q. Wu, Y. Gong, A. Wang, J.S. Alcaniz Phys. Lett. B659 (2008) 34; M.R. Setare, E. C. Vagenas Phys. Lett. B666 (2008) 111; B. Gumjudpai, T. Naskar, M. Sami, S. Tsujikawa JCAP 0506 (2005) 007.

[3] B. Wang, Y. Gong, E. Abdalla, Phys. Lett. B624 (2005) 141.

[4] J. H. He, B. Wang JCAP 0806 (2008) 010; C. Feng, B. Wang, E. Abdalla, R.-K. Su Phys. Lett. B665 (2008) 111; J. H. He, B. Wang and E. Abdalla, Phys. Lett. B 671 (2009), 139.

[5] Mubasher Jamil, Muneer Ahmad Rashid Eur. Phys. J. C56 (2008) 429, C58 (2008) 111; M.R. Setare, Elias C. Vagenas, arXiv:0704.2070; Xi-ming Chen, Yungui Gong, Emmanuel N. Saridakis, arXiv:0812.1117; Z. K. Guo, N. Ohta and S. Tsujikawa, Phys. Rev. D76 (2007) 023508; Orfeu Bertolami, F. Gil Pedro, M. Le Delliou, Phys. Lett. B654 (2007) 165; O. Bertolami, F.Gil Pedro, M.Le Delliou, arXiv:0705.3118.

[6] E. Abdalla, B. Wang Phys. Lett. B651 (2007) 89.

[7] B. Wang, J. Zang, C.-H. Lin, E. Abdalla, S. Micheletti, Nucl. Phys. B778 (2007) 69.

[8] A. Sen JHEP 04 (2002) 048; A. Sen JHEP 07 (2002) 065; A. Sen Mod. Phys. Lett. A17 (2002) 1797.

[9] T. Padmanabhan Phys. Rev. D66 (2002) 021301(R); A. Feinstein Phys. Rev. D66 (2002) 063511.

[10] J. S. Bagla, H. K. Jassal and T. Padmanabhan Phys. Rev. D67 (2003) 063504. 
[11] L. R. Abramo and F. Finelli Phys. Lett. B575 (2003) 165.

[12] B. Wang, C. Y. Lin, E. Abdalla Phys. Lett. B637 (2006) 357; J. Shen, B. Wang, E. Abdalla, R. K. Su Phys. Lett. B609 (2005) 200.

[13] L. Amendola Phys. Rev. D62 (2000) 043511; R. Bean, E. Flanagan, I. Laszlo, M. Trodden Phys. Rev. D78 (2008) 123514.

[14] G. Olivares, F. Atrio-Barandela, D. Pavon Phys. Rev. D71 (2005) 063523; G. Olivares, F. Atrio-Barandela, D. Pavon, AIP Conf. Proc. 841 (2005) 550.

[15] L. Amendola, G. C. Campos, R. Rosenfeld Phys. Rev. D75 (2007) 083506.

[16] C. Feng, B. Wang, Y. Gong, R.-K. Su JCAP 0709 (2007) 005.

[17] S. Capozziello, V. F. Cardone, M. Funaro and S. Andreon Phys. Rev. D70 (2004) 123501.

[18] R. Jimenez, L. Verde, T. Treu and D. Stern ApJ 593 (2003) 622.

[19] J. Simon, L. Verde and R. Jimenez Phys. Rev. D71 (2005) 123001.

[20] E. Komatsu, et. al. 0803.0547 [astro-ph].

[21] L. M. Krauss astro-ph/0301012.

[22] R. Cayrel, et. al. Nature 409 (2001) 691.

[23] Y. Wang, P. Mukherjee Phys. Rev. D76 (2007) 103533.

[24] G. Hinshaw, et. al. Astrophys. J. Suppl. 170 (2007) 288; D. N. Spergel, et. al. Astrophys. J. Suppl. 170 (2007) 377.

[25] D. J. Eisenstein, et. al. ApJ 633 (2005) 560.

[26] P. Astier, et. al. Astron. Astrophys. 447 (2006) 31.

[27] A. G. Riess, et. al., ApJ 659 (2006) 98.

[28] W. L. Freedman, et. al. ApJ 553 (2001) 47.

[29] D. Pavon, B. Wang, Gen. Relativ. Grav. 41 (2009),1; arXiv:0712.0565.

[30] O. Bertolami and R. Rosenfeld arXiv:0708.1784. 